\title{
Patient-specific instrumentation improves alignment of lateral unicompartmental knee replacements by novice surgeons
}

\author{
Chin Ting Justin $\mathrm{Ng}^{1} \cdot$ Simon Newman $^{1}$ (D) $\cdot$ Simon Harris $^{1} \cdot$ Susannah Clarke ${ }^{1,2}$ • \\ Justin Cobb ${ }^{1}$
}

Received: 25 November 2016/Revised: 18 March 2017 / Accepted: 23 March 2017 /Published online: 13 May 2017

(C) The Author(s) 2017. This article is an open access publication

\begin{abstract}
Purpose Patient-specific instrumentation (PSI) has the potential to offer numerous benefits - not least of all, improved resection accuracy; but its potential has not been realised in clinical studies. An explanation may be the focus of such studies on the total knee replacement (TKR - a common procedure, with which surgeons are generally very familiar. Consequently, we sought to investigate the potential role of PSI in guiding novice surgeons to perform the more technically demanding and less familiar lateral unicondylar knee replacement (LUKR).

Methods Twelve orthopaedic trainees naive to LUKR were instructed to perform the procedure according to a preoperative plan. These were carried out on synthetic sawbones and were completed once with conventional instrumentation alone and once with the adjunct of PSI, allowing a comparison of the plan adherence achieved by the two sets of instrumentation.

Results There was a tendency for PSI to demonstrate improved plan adherence, though a statistically significant improvement was only seen in compound rotational error of the femoral implant $(p=0.004)$. PSI was, however, able to produce narrower standard deviations in the mean translational displacement of the femoral implant and also the mean rotational displacement of both implants, suggesting a higher degree of precision.
\end{abstract}

Simon Newman

snewman1@imperial.ac.uk

1 MSK Lab, Department of Orthopaedics, Imperial College London, Charing Cross Hospital, Fulham Palace Road, London W6 8RF, UK

2 Embody Ltd, Imperial College London, Charing Cross Hospital, Fulham Palace Road, London W6 8RF, UK
Conclusions Our study provides some evidence that PSI can improve the ability of novice surgeons to replicate a preoperative plan, but our results suggest the need for largerscale clinical studies to establish the role of PSI in this procedure.

Keywords Patient specific instrumentation - Lateral unicondylar knee replacement $\cdot$ Novice surgeons

\section{Introduction}

Patient-specific instrumentation (PSI) involves the production of individually designed and printed cutting guides, precisely matched to the patient's own anatomy. Proposed benefits of this technology to arthroplasty surgeons include: increased accuracy of implant placement, reduced costs and faster operating times [1].

The lateral unicompartmental knee replacement (LUKR) is a relatively uncommon procedure; numbers are not recorded by most registries, including the England and Wales National Joint Registry [2], but the Swedish registry reported only three were performed in 2013 [3]. However, there is increasing evidence for the superior efficacy and lower cost of unicompartmental knee replacement (UKR) over TKR in cases of single compartment arthrosis [4-8]. Importantly, there is also evidence of a better safety profile with this smaller operation, with lower rates of morbidity and mortality [9]. Despite these benefits, a significant barrier to increased use of UKR and LUKR in particular is the technical difficulty of the operation, a significant learning curve, and the need to perform larger numbers to achieve the lowest reoperation rates, which have traditionally been higher in UKR compared with TKR surgery $[10,11]$. As a means of potentially simplifying and improving the accuracy of a procedure, PSI may 
offer a way to increase the use of the LUKR and allow more patients to experience this small, safe and effective operation.

Seeking to test this potential, we assessed the accuracy of placement of LUKR implants by orthopaedic surgical trainees unfamiliar with the operation. Through the analysis of synthetic bones used on a LUKR training course, where both PSI and conventional instrumentation were used, we compared the adherence of the trainee surgeons to a specified operative plan using each technique. Our null hypothesis was that there would be no difference in the degree of deviation from the plan using either technique.

\section{Material and methods}

Twelve orthopaedic trainees attended an instructional course on LUKR. None of the trainees had practical experience of the procedure and, as part of the course, performed their first two LUKR procedures on synthetic dry bones (Biomet, Bridgend, UK). All the synthetic bones were identical and were developed from the computed tomography (CT) scan of a patient with knee osteoarthritis. They consisted of a femur and a tibia joined by tight cords that replicated the collateral and cruciate ligaments.

Prior to performing the LUKRs, all the surgeons were privy to a live demonstration of the procedure using both PSI and conventional instrumentation and were also presented with an instructional booklet, which included detailed diagrams and measurements of the surgical plan. This plan was created by an experienced surgeon on in-house planning software and was designed to recreate the natural joint line using implant positions that could be realistically achieved with conventional instrumentation. PSI guides were designed and produced by Embody (London, UK) using the EOS Formiga P110 Printing System with a medical grade polymer (PA2200) (Figs. 1 and 2).

Each trainee was then charged with performing the LUKR twice: once with PSI (Embody, London, UK) and once with conventional instrumentation (Oxford Phase 3 Instrumentation, Biomet, Bridgend UK). Half the surgeons used PSI first and the other half used conventional instrumentation before swapping over. Trainees were guided individually by experienced faculty and asked to replicate the preoperative plan.

After the resections were complete, three-dimensionally printed simplified femoral and tibial LUKR implants were glued onto the bones. The implants included multiple reference spikes that were used during the process of registering the implant position relative to the bone (Figs. 3 and 4). An investigator who was blinded to the instrumentation used to prepare the implant beds completed the placement of the implants onto the cut surfaces.

A desktop laser scanner (NextEngine 3D, Santa Monica, CA, USA) was used to create three-dimensional

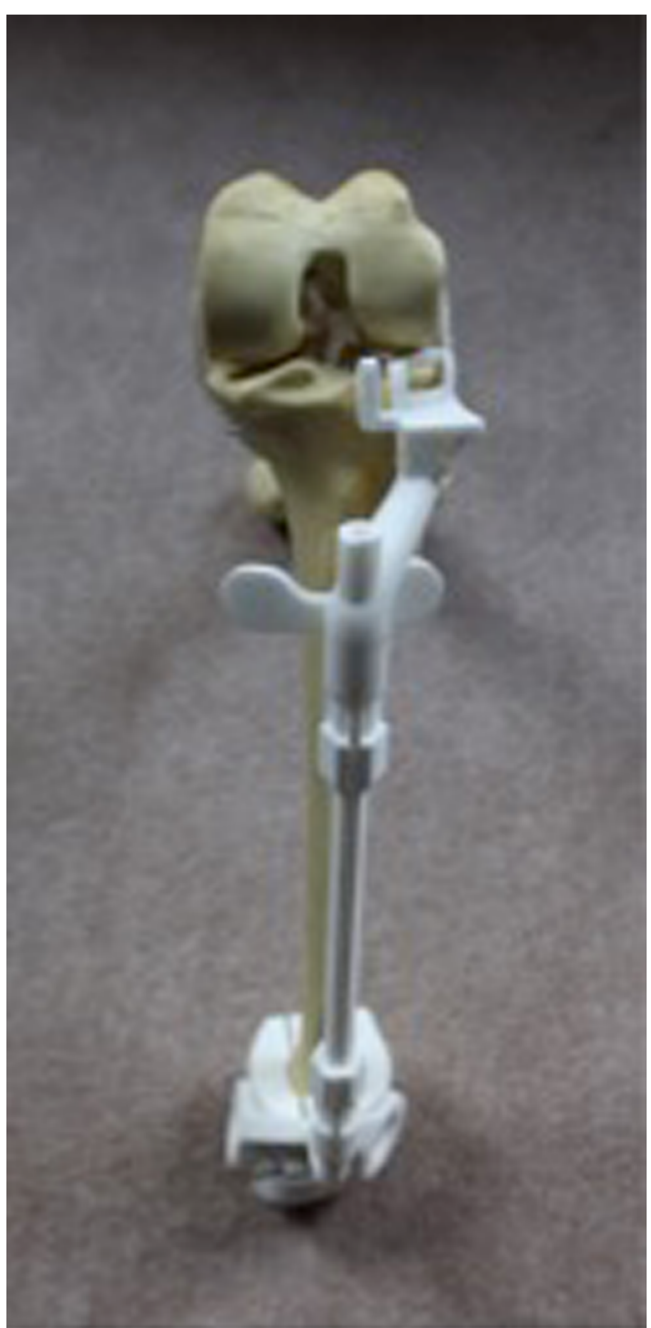

Fig. 1 Tibial PSI guide

representations of the cut bones and their accompanying implants. The scanner has a reported accuracy to within $0.127 \mathrm{~mm}$ and has also been validated in previous studies to contain a root mean square (RMS) error of less than $1 \mathrm{~mm}$ compared with a pre-operative CT plan [12]. The scanned images were formatted as stereolithograph (STL) files and transferred into in-house planning software to identify predetermined reference points on the positioned implants.

Once the reference points were identified, they allowed investigators (who were blinded to the method of instrumentation) to determine the extent to which the positioned implants deviated from the predetermined plan. This was presented in terms of compound rotational and translational errors and also as the displacement in each of the six degrees of freedom. Compound rotational error was calculated by finding the sum of the RMS values for axial rotation, flexionextension and varus-valgus and compound translational error was calculated by finding the sum of the RMS values for absolute anterior-posterior, medial-lateral and proximal-distal translations. 


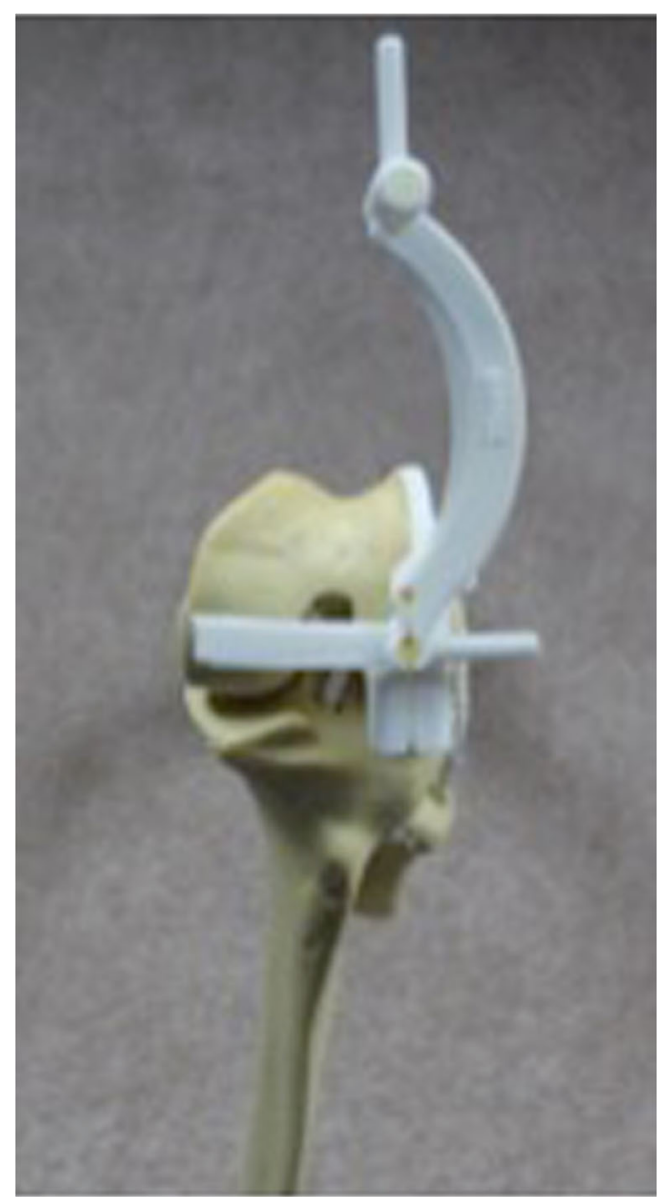

Fig. 2 Femoral PSI guide

The referencing stage of the method was repeated to validate the accuracy of our methodology. Intra-observer reliability was assessed by re-referencing the same bone five times on separate days and the mean rotational and translational errors compared. Inter-observer reliability was assessed by re-referencing four separate bones from which Bland-Altman plots were created.

Statistical analysis was performed using Statistical Package for the Social Sciences (SPSS 22, IBM, New York, NY, USA), with statistical significance set at $p<0.05$. Visual assessment of histograms and the Kolmogorov-Smirnov test confirmed normality of distribution of our data and a paired $t$-test was used for analysis with Holm's sequential Bonferroni correction to accommodate multiple comparisons.

No human or animal tissue was used during this study.

\section{Results}

\section{Tibial and femoral compound error}

There was a trend for lower measures of mean compound error in the PSI group compared to conventional instrumentation (Fig. 5) in both the tibia and femur with respect to translation and rotation. However, statistical significance was only achieved with respect to femoral rotational $(p=0.004)$.

\section{Displacement across the six degrees of freedom}

There was no significant improvement in any degree of freedom between the two forms of instrumentation, though there was a strong trend for improvement in tibial resection depth accuracy with PSI (Fig. 6). There was a significant difference between the errors achieved for medial and lateral translation, with the PSI guides producing excessive medialisation, whilst the conventional instrumentation produced excessive lateralisation. The spread of errors achieved by trainees, represented by the standard deviation, were almost universally narrower with PSI across the six degrees of freedom, and when calculated, the mean standard deviations for rotational and translational displacements for both implants were lower (Tables 1 and 2), denoting a higher level of precision with PSI.

\section{Reliability studies}

Both inter- and intra-observer studies and their accompanying Bland-Altman plots demonstrated values that were within \pm 1.96 standard deviations of the mean difference and thereby within acceptable limits of agreement.

\section{Discussion}

LUKR is a smaller, safer, cheaper and more effective operation than TKR for the treatment of lateral compartment arthrosis $[5,13]$. On this basis LUKR should be a popular procedure. However, although isolated lateral compartment disease affects $10 \%$ of patients, LUKR accounted for only three out of 493 UKRs from a total of 13,338 knee arthroplasties in the

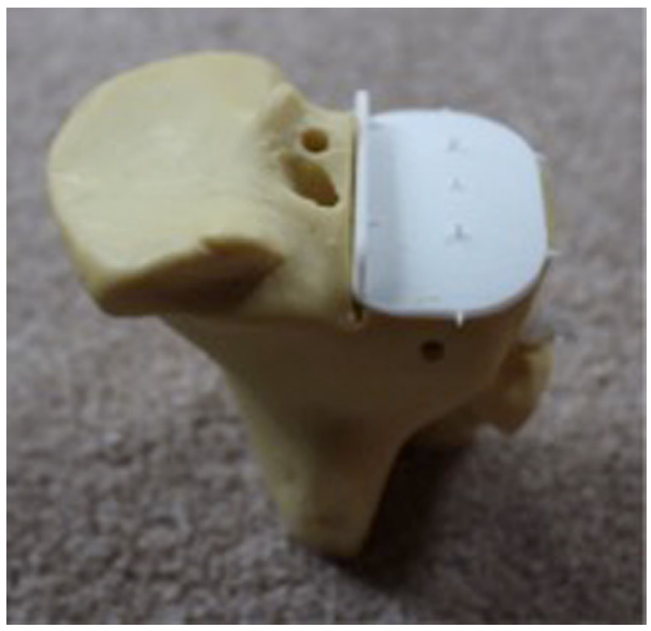

Fig. 3 Simplified implant with reference spikes in situ on prepared tibia 


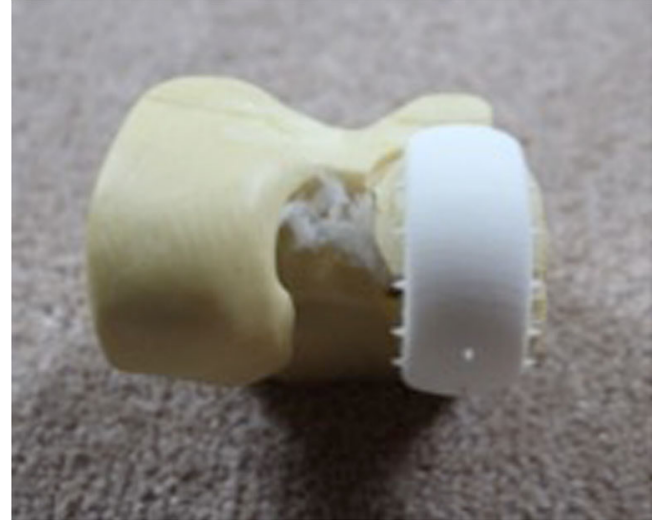

Fig. 4 Simplified implant with reference spikes in situ on prepared femur

2014 Swedish registry report $[3,14]$. There are a number of reasons for the reluctance of surgeons to use UKR and LUKR in particular: improved function comes at the cost of a higher reoperation rate - a fact that is heavily publicised through registry reports $[2-4,15]$. In the United Kingdom this has extended to the publication of unit-level data by the National Health Service and to the reporting of surgeons who are outliers with respect to revision rate for knee arthroplasty to their hospital executives [2]. UKR surgery is also associated with a learning curve that leads to poorer results whilst acquiring experience of the procedure [16, 17]; it can, consequently, be difficult for surgeons to learn on their patients rather than use the more familiar TKR. LUKR is a technically challenging operation due to difficulty of obtaining adequate exposure and lateral collateral ligament laxity in flexion, making gap balancing more complicated than in medial UKR [11].

Logic suggests that PSI should improve implant positioning and our experience has been that complex operations, in particular, can be made simpler with the assistance of patient matched guides. However, studies to date, principally on the use of PSI guides in TKR surgery, have failed to show significant clinical improvements, particularly with respect to component alignment. An issue with the existing literature is that studies assessing PSI are dominated by TKR procedures that are facilitated by generally well-designed conventional instrumentation and performed by surgeons experienced in the procedure. It has been suggested that the benefits of PSI may instead be felt most by inexperienced surgeons performing
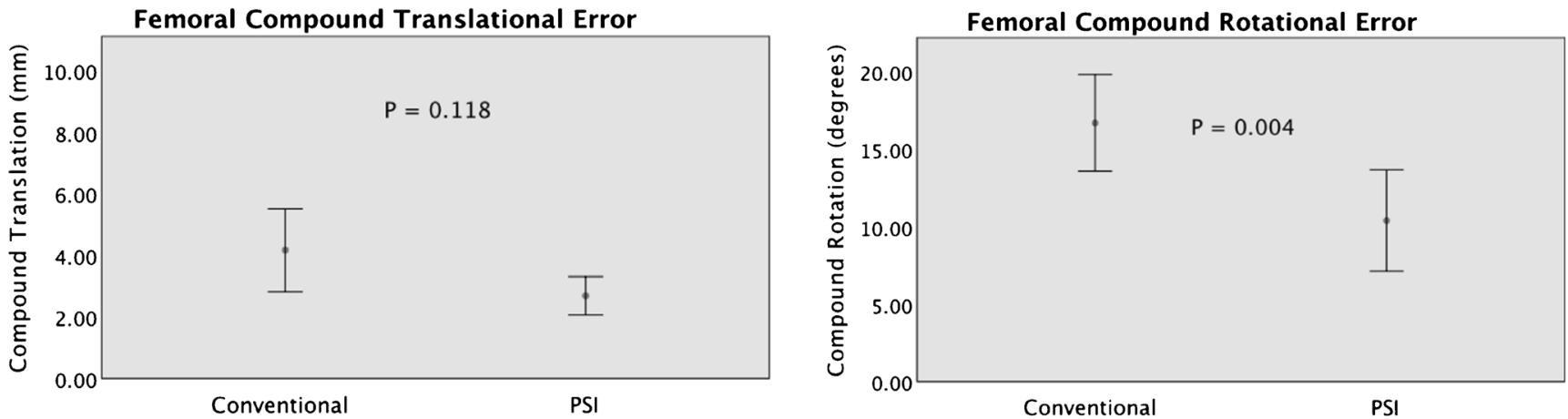

Tibial Compound Translational Error
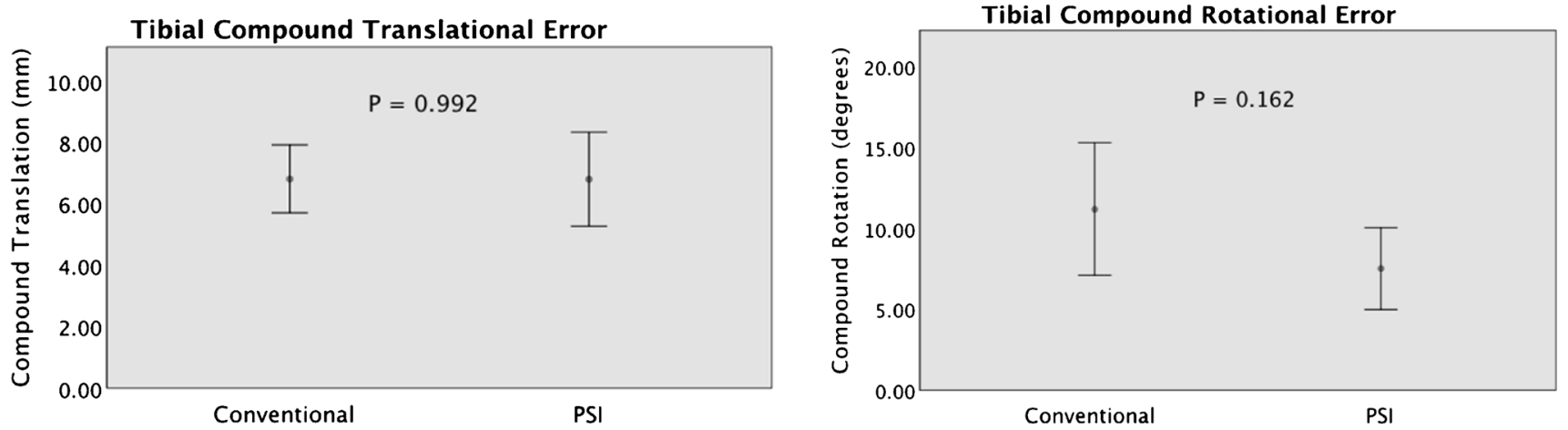

Fig. 5 Femoral and tibial compound translational and rotational error expressed as interval plots: mean \pm 2 standard errors of the mean 

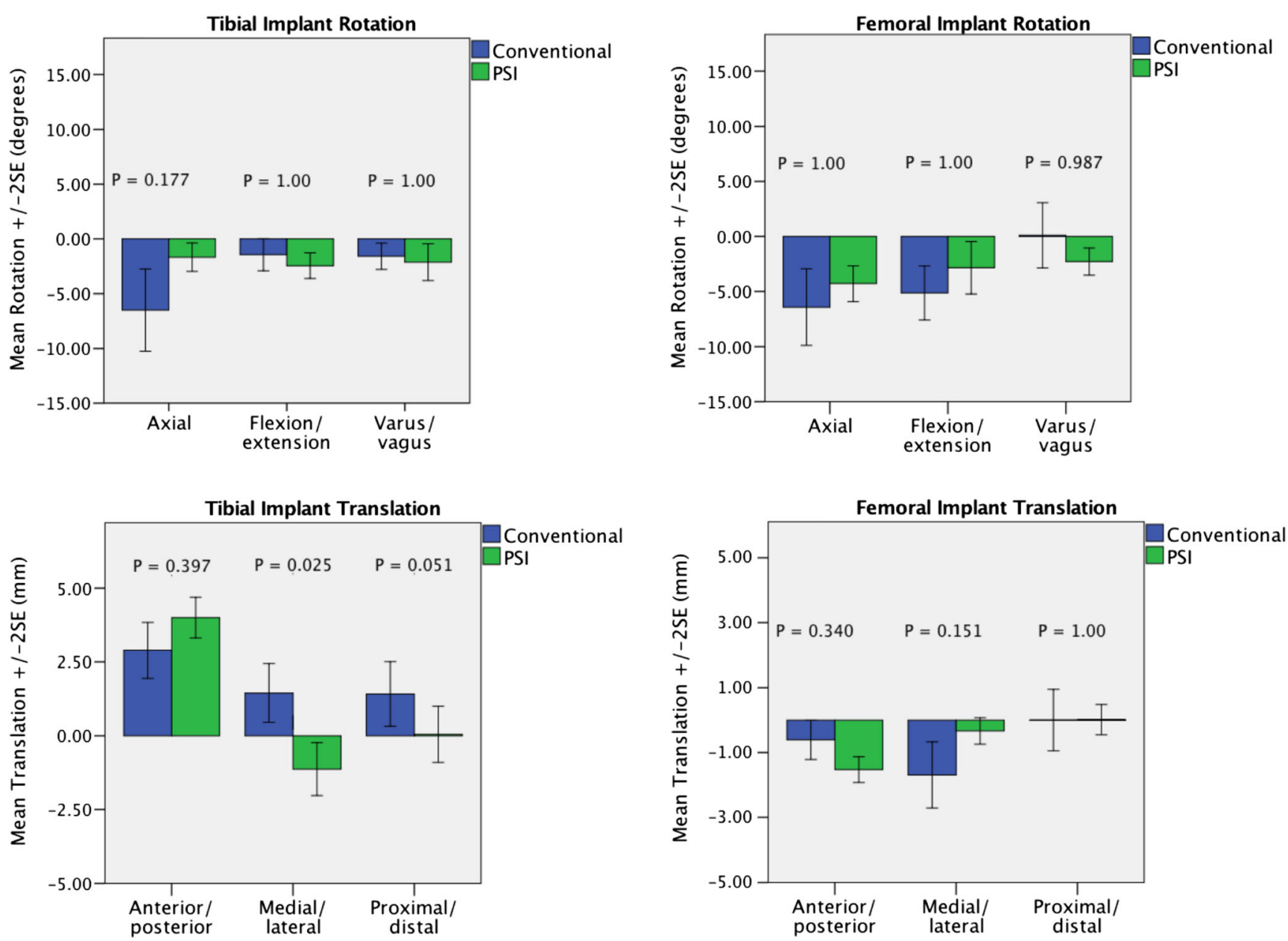

Fig. 6 Implant positioning errors for each of the six degrees of freedom represented as mean rotational and translational deviations \pm 2 standard errors of the mean

unfamiliar procedures [18]. PSI therefore offers a potential way to reduce the technical challenge and shorten the learning curve of the effective but underutilised LUKR. This may help surgeons feel more confident to perform the procedure and reduce the fear driven by the registry reports. Our study sought to investigate the benefits of PSI with this operation when performed by inexperienced surgeons. The results demonstrated non-inferiority of PSI and showed some evidence that improvements may be made in accuracy of implant placement with PSI over conventional instruments.

In particular, this study demonstrated an improvement in femoral implant compound rotation and a trend for
Table 1 Standard deviations of tibial implant positional error (values to nearest millimetres or degree)

\begin{tabular}{llll}
\hline Degrees of freedom & PSI & Conventional instrumentation & Difference \\
\hline Axial rotation $\left({ }^{\circ}\right)$ & 2 & 7 \\
Flexion-extension $\left(^{\circ}\right)$ & 2 & 3 \\
Varus-valgus $\left({ }^{\circ}\right)$ & 3 & 2 & 2 \\
Mean rotational error standard deviation $\left({ }^{\circ}\right)$ & 2 & 4 & \\
Anterior-posterior $(\mathrm{mm})$ & 1 & 1 & \\
Medial-lateral $(\mathrm{mm})$ & 2 & 2 & 0 \\
Proximal-distal $(\mathrm{mm})$ & 2 & 2 & \\
Mean translational error standard deviation $(\mathrm{mm})$ & 2 & 2 &
\end{tabular}


Table 2 Standard deviations of femoral implant positional error (values rounded to nearest millimetre and degree)

\begin{tabular}{llll}
\hline Degrees of freedom & PSI & Conventional Instrumentation & Difference \\
\hline Axial rotation $\left({ }^{\circ}\right)$ & 3 & 6 & \\
Flexion-extension $\left({ }^{\circ}\right)$ & 4 & 4 & \\
Varus-valgus $\left({ }^{\circ}\right)$ & 2 & 5 & 2 \\
Mean rotational error standard deviation $\left(^{\circ}\right)$ & 3 & 5 & \\
Anterior-posterior (mm) & 1 & 1 & 1 \\
Medial-lateral (mm) & 1 & 2 & \\
Proximal distal (mm) & 1 & 2 & \\
Mean translational error standard deviation $(\mathrm{mm})$ & 1 & 2 & \\
\hline
\end{tabular}

improvement in tibial rotation and femoral translational compound errors. Levels of precision, as measured by the standard deviation of the errors measured, were greater for the PSI procedures than with the conventional instrumentation. We were unable to demonstrate any significant improvement in the individual degrees of freedom for either tibial or femoral implant placement, though there was a strong trend for improvement with PSI with respect to implant resection depth. There was a difference between the placement of the tibial implants in the coronal plane, with PSI resulting in greater medialisation of the implant and conventional instrumentation tending towards lateralisation. This was due to the tightness of the cord ligaments making placement of the sagittal saw cut in the planned position difficult; importantly, PSI tended to result in the medialisation of the tibial implant, which reduces the risk of bearing dislocation compared with a more laterally placed implant that could lead to the bearing impinging on the medial tibial implant wall [19].

The benefits of PSI have been questioned in the literature, but these conclusions have predominantly been based upon the assessment of experienced surgeons undertaking a common procedure, specifically TKR [1]. This has potentially undervalued the benefits of PSI with infrequently performed procedures, especially when undertaken by inexperienced surgeons.

There is sparse literature on the clinical use of PSI in UKR surgery. With respect to LUKR, Demange et al. [20] described the use of PSI in the placement of custom-made LUKR implants compared with a retrospective series of conventionally sited Miller-Galante implants. Whilst this study reported superior survivorship and greater tibial plateau coverage with the custom implants, the study does not specifically address the question of whether PSI is helpful in LUKR surgery. With respect to the use of PSI in medial UKR (MUKR) surgery, Trong et al. [21] reported excellent implant alignment when comparing patients' post-operative CTs to pre-operative plans. Kerens et al. [22] found that PSI improved the position of the femoral component in the frontal plane, but had no effect on other implant placement parameters. However, Ollivier et al. [23] found no differences in functional score, gait, lower limb alignment or implant positioning between PSI and conventional instrumentation.
There are limitations to this study. The degree of implant placement superiority demonstrated by PSI over conventional instrumentation was less than might have been expected. This may be attributed to underpowering of the study. However, a sample size of 12 has been advocated as a suitable size for studies such as this, where there are insufficient data for an a priori power calculation [24] and previous in vitro studies conducted on MUKR have used similar numbers of subjects [25]. A greater improvement with PSI may also have been observed with a higher-fidelity simulation, such as using a cadaver. This is because whilst conventional instrumentation relies heavily on landmarks (which were clearly seen with our synthetic bone model) to maintain its accuracy, PSI is less likely to benefit from this increased visibility. However, the use of synthetic bones is well established in arthroplasty research [12] and allowed greater consistency between trials than could have been achieved with cadaveric specimens.

In conclusion, this study demonstrates that PSI can lead to some improvement in the accuracy of replicating an operative plan by novice LUKR surgeons. The effect demonstrated in this study is modest and further investigation is required to determine whether these improvements are translated into improved in vivo LUKR implant positioning as well as patient outcomes. However, our study would suggest PSI may have the potential to assist inexperienced surgeons undertaking the effective, but underutilised LUKR.

Acknowledgements We are grateful to all the orthopaedic trainees who took part in the course and to Biomet UK for supplying the synthetic bones and conventional instrumentation.

\section{Compliance with ethical standards}

Conflict of interest JC and SC are directors of a company (Embody Ltd) that produces PSI.

Open Access This article is distributed under the terms of the Creative Commons Attribution 4.0 International License (http:// creativecommons.org/licenses/by/4.0/), which permits unrestricted use, distribution, and reproduction in any medium, provided you give appropriate credit to the original author(s) and the source, provide a link to the Creative Commons license, and indicate if changes were made. 


\section{References}

1. Thienpont E, Schwab PE, Fennema P (2014) A systematic review and meta-analysis of patient-specific instrumentation for improving alignment of the components in total knee replacement. Bone Joint J 96-B(8):1052-1061. doi:10.1302/0301-620X.96B8.33747

2. Wishart N, Beaumont R, Young E, Mccormack V, Swanson M (2014) National Joint Registry for England, Wales, Northern Ireland, and Isle of Man 11th Annual Report. http://www. njrcentre.org.uk/njrcentre/Portals/0/Documents/England/Reports/ 11 th_annual_report/NJR\%2011 th\%20Annual\%20Report\% 202014.pdf. Accessed 13 March 2017

3. Sundberg M, Lidgren L, W-Dahl A, Robertsson O (2014) Swedish Knee Arthroplasty Register Annual Report. http://www.myknee.se/ pdf/SKAR2014_Eng_1.1.pdf. Accessed 13 March 2017

4. Rothwell A, Larmer P, Hobbs T, Rothwell A (2014) The New Zealand Joint Registry Annual Report. http://nzoa.org.nz/system/ files/Web_DH7657_NZJR2014Report_v4_12Nov15.pdf. Accessed 13 March 2017

5. Liddle AD, Pandit H, Judge A, Murray DW (2015) Patient-reported outcomes after total and unicompartmental knee arthroplasty: a study of 14,076 matched patients from the National Joint Registry for England and Wales. Bone Joint J 97-B(6):793-801. doi:10. 1302/0301-620X.97B6.35155

6. Wiik AV, Manning V, Strachan RK, Amis AA, Cobb JP (2013) Unicompartmental knee arthroplasty enables near normal gait at higher speeds, unlike total knee arthroplasty. J Arthroplast 28(9): 176-178. doi:10.1016/j.arth.2013.07.036

7. Hopper GP, Leach WJ (2008) Participation in sporting activities following knee replacement: Total versus unicompartmental. Knee Surg Sports Traumatol Arthrosc 16(10):973-979. doi:10. 1007/s00167-008-0596-9

8. Willis-Owen CA, Brust K, Alsop H, Miraldo M, Cobb JP (2009) Unicondylar knee arthroplasty in the UK National Health Service: an analysis of candidacy, outcome and cost efficacy. Knee 16(6): 473-478. doi:10.1016/j.knee.2009.04.006

9. Liddle AD, Judge A, Pandit H, Murray DW (2014) Adverse outcomes after total and unicompartmental knee replacement in 101 330 matched patients: a study of data from the National Joint Registry for England and Wales. Lancet 384(9952):1437-1445. doi:10.1016/S0140-6736(14)60419-0

10. Hamilton WG, Ammeen D, Engh CA, Engh GA (2010) Learning curve with minimally invasive unicompartmental knee arthroplasty. J Arthroplast 260(1):37-45. doi:10.1016/j.arth.2009.05.011

11. Servien E, Merini A, Lustig S, Neyret P (2013) Lateral unicompartmental knee replacement: current concepts and future directions. Knee Surg Sports Traumatol Arthrosc 21(11):2501-2508. doi:10.1007/s00167-013-2585-x

12. Jaffry Z, Masjedi M, Clarke S, Harris S, Karia M, Andrews B et al (2014) Unicompartmental knee arthroplasties : robot vs patient specific instrumentation. Knee 21(2):428-434. doi:10.1016/j. knee.2013.11.017

13. Ollivier M, Abdel MP, Parratte S, Argenson JN (2014) Lateral unicondylar knee arthroplasty (UKA): contemporary indications, surgical technique, and results. Int Orthop 38(2):449-455. doi:10. 1007/s00264-013-2222-9

14. Ahlbäck S (1968) Osteoarthrosis of the knee. A radiographic investigation. Acta Radiol Diagn (Stockh) Suppl 277:7-72

15. Batten J, Steiger RD, Mercer G (2014) Australian National Joint Registry Annual Report. 2014

16. Rees JL, Price AJ, Beard DJ, Dodd C, Murray DW (2004) Minimally invasive Oxford unicompartmental knee arthroplasty: functional results at 1 year and the effect of surgical inexperience. Knee 11(4):363-367. doi:10.1016/j.knee.2003.12.006

17. Zhang Q, Zhang Q, Guo W, Liu Z, Cheng L, Yue D et al (2014) The learning curve for minimally invasive Oxford phase 3 unicompartmental knee arthroplasty: cumulative summation test for learning curve (LC-CUSUM). J Orthop Surg Res 9(1):81. doi: 10.1186/s13018-014-0081-8

18. Bell SW, Stoddard J, Bennett C, London NJ (2014) Accuracy and early outcomes in medial unicompartmental knee arthroplasty performed using patient specific instrumentation. Knee 21(1):S33S36. doi:10.1016/S0968-0160(14)50007-5

19. Weston-Simons JS, Kendrick BJL, Mentink MJA, Pandit H, Gill HS, Murray DW (2014) An analysis of dislocation of the domed Oxford lateral Unicompartmental knee replacement. Knee 21(1): 304-309. doi:10.1016/j.knee.2013.04.008

20. Demange MK, Von Keudell A, Probst C, Yoshioka H, Gomoll AH (2015) Patient-specific implants for lateral unicompartmental knee arthroplasty. Int Orthop 39(8):1519-1526. doi:10.1007/s00264015-2678-x

21. Trong MLD, Diezi C, Goerres G, Helmy N (2015) Improved positioning of the tibial component in unicompartmental knee arthroplasty with patient-specific cutting blocks. Knee Surg Sports Traumatol Arthrosc 23(7):1993-1998. doi:10.1007/s00167-0142839-2

22. Kerens B, Schotanus MGM, Boonen B, Kort NP (2015) No radiographic difference between patient-specific guiding and conventional Oxford UKA surgery. Knee Surg Sports Traumatol Arthrosc 23(5):1324-1329. doi:10.1007/s00167-014-2849-0

23. Ollivier M, Parratte S, Lunebourg A, Viehweger E, Argenson JN (2016) The John Insall award: No functional benefit after unicompartmental knee arthroplasty performed with patientspecific instrumentation: a randomized trial. Clin Orthop Relat Res 474(1):60-68. doi:10.1007/s11999-015-4259-0

24. Julious SA (2005) Sample size of 12 per group rule of thumb for a pilot study. Pharm Stat 4(4):287-291. doi:10.1002/pst.185

25. Heyse TJ, Lipman JD, Imhauser CW, Tucker SM, Rajak Y, Westrich GH (2014) Accuracy of individualized custom tibial cutting guides in UKA. HSS J 10(3):260-265. doi:10.1007/s11420$014-9410-z$ 